\title{
AC Impedance Measurement and Electron Holography of Ionic Liquid in a Transmission Electron Microscope
}

\author{
Yoh Iwasaki $^{1, *}$, Ken Harada ${ }^{1}$, Keiko Shimada $^{1}$, Manabu Shirai ${ }^{2}$ and Daisuke Shindo ${ }^{1,3}$ \\ ${ }^{1}$ Center for Emergent Matter Science, RIKEN, c/o Hitachi Center for Exploratory Research, Hatoyama, Saitama 350-0395, Japan \\ ${ }^{2}$ Science Systems Product Div., Hitachi High-Technologies Corporation, Hitachinaka 312-0033, Japan \\ ${ }^{3}$ Institute of Multidisciplinary Research for Advanced Materials, Tohoku University, Sendai 980-8577, Japan
}

\begin{abstract}
Alternating current (AC) impedance measurements were performed with ionic liquid (IL) specimens to study feasibility of in-situ characterization method for conductive and dielectric properties of materials in a transmission electron microscope (TEM). A bridge-shaped IL specimen was prepared between two molybdenum (Mo) probe tips in a TEM, and was exposed to electron beam. The repeated AC impedance measurements during the irradiation revealed that the conductance of the IL bridge decreased faster at higher frequencies. Electron holography around the IL before and after the electron irradiation revealed enhanced charging after the irradiation, which is consistent with the loss of conductance by the irradiation. AC impedance measurement and electron holography are compatible with TEM to complementarily provide dynamic transport properties and potential distribution of a specimen. [doi:10.2320/matertrans.MI201903]
\end{abstract}

(Received September 4, 2019; Accepted December 18, 2019; Published January 31, 2020)

Keywords: electron microscopy, in-situ observation, impedance measurement, dielectric properties, electron holography, ionic liquid

\section{Introduction}

In situ or operando measurements have become popular in materials science. Various types of measurements including the electrical, magnetic or mechanical properties of materials have also been made with microscopic imaging in electron microscopes. ${ }^{1-8)}$ For the characterization of electronic properties, information on both the voltage and current through the specimen is invaluable. Alternating current (AC) impedance measurement is a popular method in characterizing conducting or dielectric properties of materials. However, impedance measurement becomes more difficult when the measurement current becomes smaller with the dimension of the specimen. In this study, we have tried to demonstrate $\mathrm{AC}$ impedance measurements and electric potential measurements by electron holography on specimens in a transmission electron microscope (TEM).

An ionic liquid (IL) was adopted as the object for characterization. ILs are recognized as a class of functional material with a wide variety of applications such as electrolytes in batteries. ${ }^{9-14)}$ The IL can be stably stored in a vacuum due to its low vapor pressure. In electron microscopy, ILs are known as powerful substances that help prevent specimens from electrostatic charging, due to their own conductivity. ${ }^{15)}$ However, a loss of conductivity over time has been observed under exposure of the IL to electrons. ${ }^{16)}$ In this report, quantitative impedance monitoring of an IL specimen during electron irradiation is demonstrated, and the results are compared with electron holography.

\section{Experimental Procedure}

A TEM with an acceleration voltage of $300 \mathrm{kV}$ (HF3300X, Hitachi High-Technologies Co.) was used in this experiment. A specimen holder equipped with a probe needle with threeaxes of motion was fabricated by Sunyou Co. Each axis of

*Corresponding author, E-mail: yoh.iwasaki@riken.jp motion is driven by both a mechanical screw and a piezoelectric device for coarse and fine positioning, respectively.

\subsection{AC impedance measurement}

AC impedance measurements, or impedance spectroscopy, is a method of characterizing dielectric properties, and has many applications for electrochemical processes. ${ }^{17-19)}$ The measurement system used in this study is schematically illustrated in Fig. 1. The sinusoidal signal from a function generator was introduced into a TEM via an electrical feedthrough on the specimen holder, supplied to a metal needle probe tip, and then applied to the IL specimen that was bridging to another metallic tip electrically grounded to the microscope.

The electrical resistance of a small IL specimen is high; therefore, direct measurement of the IL specimen alone with DC or at low frequency would have a very low signal-tonoise ratio. By means of a moderately high (ca. 2M 2 ) resistor, $\mathrm{R}_{\mathrm{EXT}}$, added to the circuit, a subtle change in the high impedance of the IL can be sensitively reflected to the change in phase of the current signal with frequency, $f$, around $2 \pi f=1 / \mathrm{R}_{\text {EXT }} \mathrm{C}_{\text {STRAY }}$.

The current flowing through the IL, $\mathrm{R}_{\mathrm{EXT}}$, and $\mathrm{C}_{\text {STRAY }}$ was measured via the voltage across a shunt resistor, $\mathrm{R}_{\mathrm{SHUNT}}$, placed between the function generator and the specimen. The amplitude and phase of the current and voltage signal were both measured with a lock-in amplifier. The impedance, $\mathrm{Z}_{\text {HOLDER }}(f)$, of the specimen holder of the TEM including IL, $\mathrm{R}_{\text {EXT }}$ and $\mathrm{C}_{\mathrm{STRAY}}$ is a complex number defined at each measurement frequency, $f$, as a ratio of the voltage and current signals, i.e. $\mathrm{Z}_{\mathrm{HOLDER}}(f)=\mathrm{V}(f) / \mathrm{I}(f)$. The impedance for the IL only, $\mathrm{Z}_{\mathrm{IL}}(f)$, was then derived via subtraction of conductance of parallel components as $1 /$ $\mathrm{Z}_{\mathrm{IL}}(f)=1 / \mathrm{Z}_{\mathrm{HOLDER}}(f)-1 / \mathrm{Z}_{\mathrm{BLANK}}(f)$, where $\mathrm{Z}_{\mathrm{BLANK}}(f)$ is the impedance of $\mathrm{R}_{\mathrm{EXT}}$ and $\mathrm{C}_{\mathrm{STRAY}}$ of the specimen holder measured without IL bridge on the holder.

The frequency range mainly used was $3 \mathrm{~Hz}$ to $100 \mathrm{kHz}$. The signal amplitude, $\pm 50 \mathrm{mV}$, from the function generator 

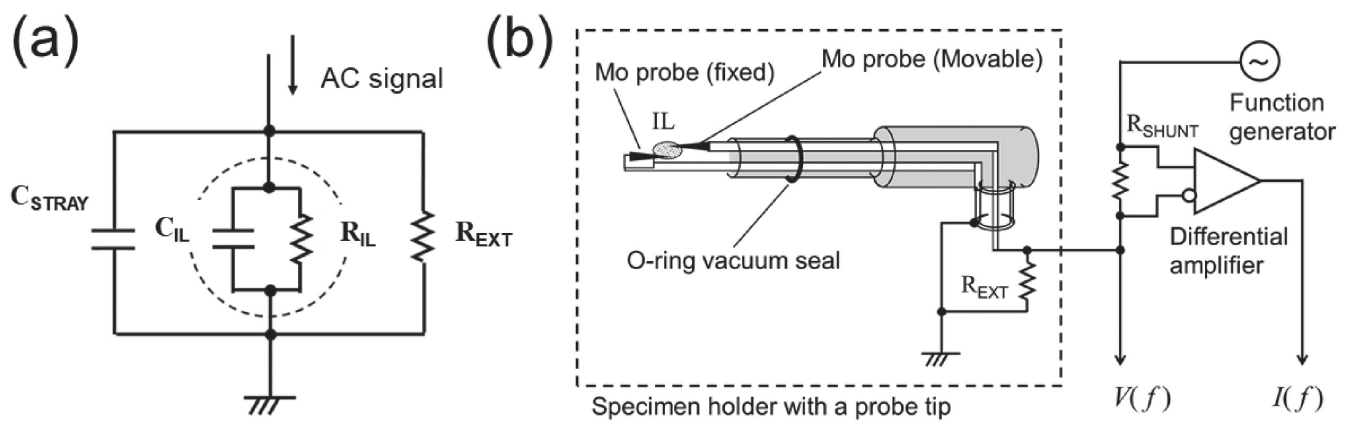

Fig. 1 (a) Equivalent circuit of the specimen holder and (b) schematic diagram of the impedance measurement setup.
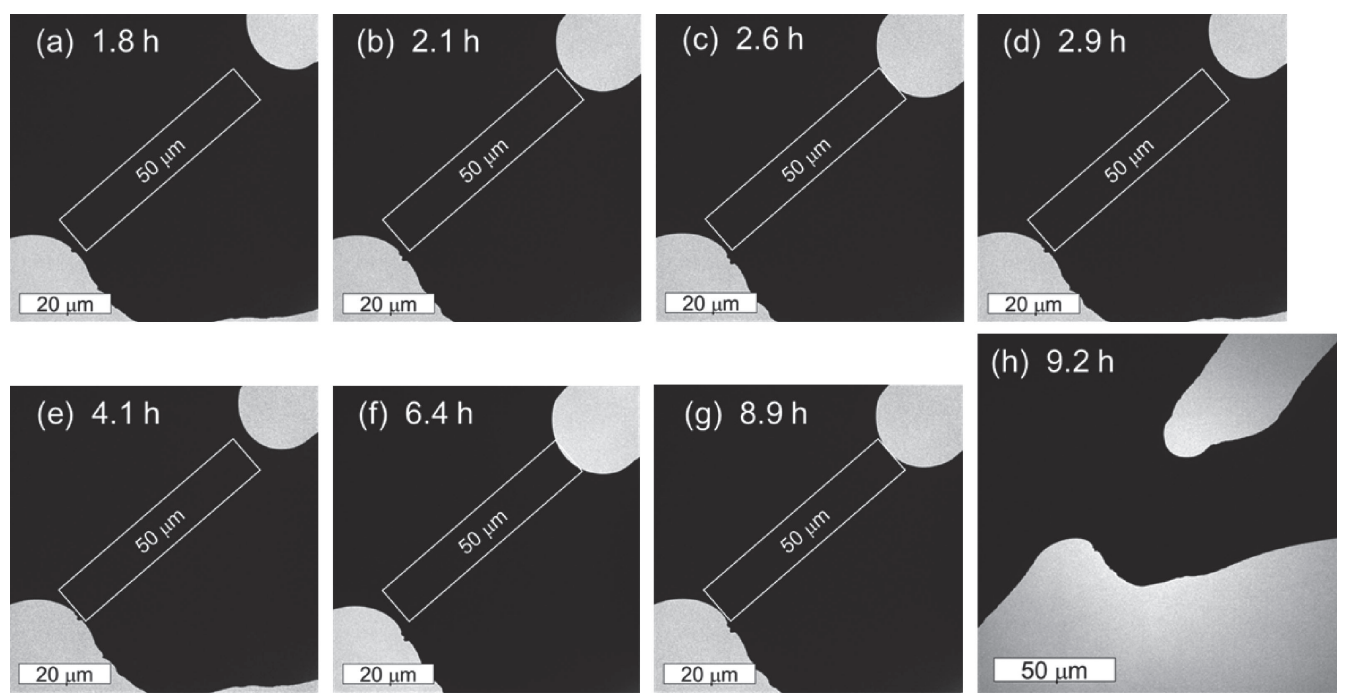

Fig. 2 Series of images of the IL bridge recorded in the course of electron irradiation in a TEM. A dark region in each image, (a) to (h), represents shadow of Mo probes (upper left and lower right) and an IL bridge supported in between. A $50 \mu \mathrm{m}$-wide white rectangle is superposed on each image, (a) to (g), around the neck of the IL bridge. Since every white rectangle is placed at the same position with respect to the frame of the image (corresponding to active area of the image sensor in the TEM), drift of the specimen or variance in size of the IL bridge can be recognized more easily by comparing its shadow with the rectangle.

was sufficiently small to prevent electrochemical reaction in the IL.

\subsection{Electron holography}

Electron holography is a method of characterizing the electromagnetic field in a TEM. ${ }^{20-22)}$ The TEM adopted in this experiment has two advantages for electron holography; a cold cathode electron gun and two stages of electron biprism. The former provides higher coherency for interference imaging, and the double biprism method provides a wider area of interference fringes free from intensity modulation by Fresnel diffraction with a filament wire of the electron biprism. ${ }^{23)}$

\subsection{Materials and preparation}

The IL used in this experiment was IL $1000^{\circledR}$ obtained from Hitachi High-Technologies Co. This is a hydrophilic IL that is provided specifically for application with electron microscopes. According to the supplier's information, its conductivity is $2.5 \mathrm{mS} / \mathrm{cm}$ with typical water content of $10 \% .^{24)}$

Probe tips that supported IL specimens were prepared from a $150 \mu \mathrm{m}$-thick molybdenum (Mo) wire. To support a bridgeshaped IL specimen, tip was prepared by beveling the end of the Mo wire with precise scissors. A thinner tip was also prepared by a focused ion beam (FIB).

\section{Experimental Results}

\subsection{AC impedance measurements}

Images of an IL bridge formed between two Mo probes are shown in Fig. 2 with accumulated time of electron irradiation indicated on each image. An IL bridge formed in air could not be introduced into the TEM without breaking the bridge. In the course of introduction of specimen into the TEM, torsion acting on the specimen holder seemed to deform geometry between the Mo probes and thus the IL bridge originally prepared in air was broken. An IL bridge had to be prepared again in the field of view of the TEM by manipulating a movable probe. It took an hour or more to stabilize drifts of specimen stage and a support for the Mo probe after the IL bridge had been rebuilt. As is recognized in Fig. 2, width (or diameter) of the bridge was smaller at $2.1 \mathrm{~h}$ and $2.6 \mathrm{~h}$ electron irradiation reflecting the instability mentioned above.

Results of AC impedance measurements are presented in terms of conductance $(1 / \mathrm{R})$ and capacitance $(\mathrm{C})$ which are obtained from real and imaginary part of complex reciprocal 


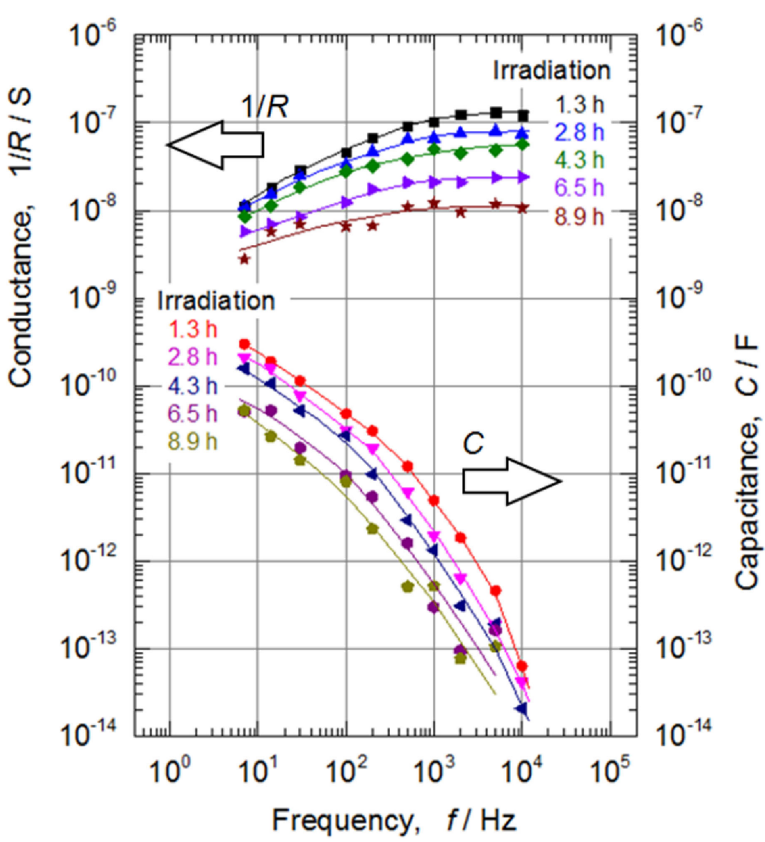

Fig. 3 Frequency dependent spectra of conductance $(1 / R)$ and capacitance (C) of an IL bridge measured after periods of electron irradiation in a TEM. Lines are drawn for guides to eye.

of the impedance respectively, i.e. $1 / Z=1 / \mathrm{R}+2 \pi j f \mathrm{C}$, where $j$ is imaginary unit and $f$ stands for frequency.

Figure 3 represents frequency dependent spectra of conductance $(1 / R)$ and capacitance (C) of the specimen. As the electron irradiation time became longer, $1 / \mathrm{R}$ and $\mathrm{C}$ spectra both shifted downward. After a long irradiation, spectra lost smoothness reflecting degraded $\mathrm{S} / \mathrm{N}$ ratio.

Time evolution of $1 / \mathrm{R}$ and $\mathrm{C}$ measured at respective frequencies is presented in Fig. 4. The $1 / \mathrm{R}$ and $\mathrm{C}$ values generally decreased with time. Pronounced scattering of data points are observed in $1 / \mathrm{R}$ at low frequency and $\mathrm{C}$ at high frequencies. A dip is observed on each plot at 2 and $2.5 \mathrm{~h}$

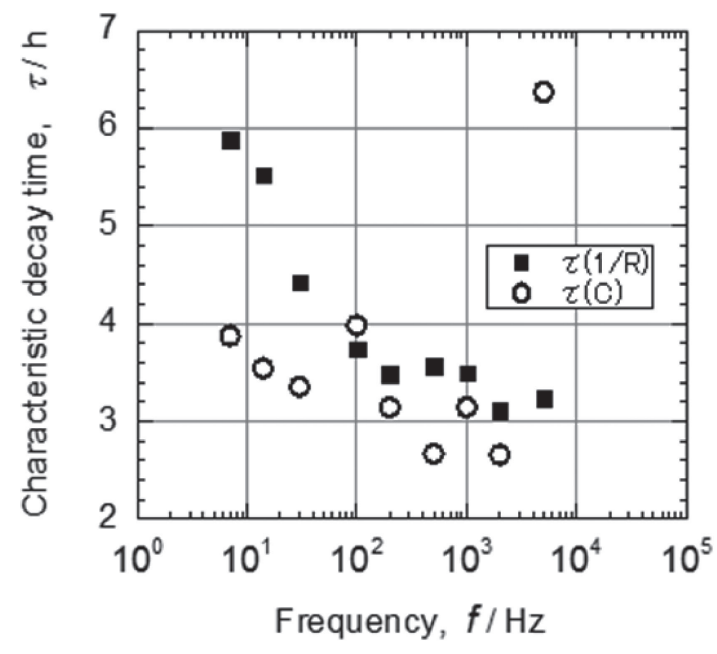

Fig. 5 Characteristic decay time, $\tau$, of $1 / \mathrm{R}$ and $\mathrm{C}$ of the IL bridge specimen measured at different frequencies.

irradiation, which reflect the narrowing of the bridge diameter noted referring to Fig. 2.

Exponential functions were fitted to plots in Fig. 4 to derive characteristic decay time for $1 / \mathrm{R}$ and $\mathrm{C}$ at respective frequencies. Obtained decay time constants, $\tau$ 's, are summarized in Fig. 5. Decay time constants for capacitance, C, are distributed around 3 to 4 hours except for $5 \mathrm{kHz}$ where pronounced scattering was observed in Fig. 4(b). Decay time constants for conductance, $1 / \mathrm{R}$, showed clear trend; $1 / \mathrm{R}$ at higher frequencies decay faster than $1 / \mathrm{R}$ at lower frequencies.

AC impedance measurements of the IL were performed also with different specimen geometries (bulk droplet and bridges) for comparison. Obtained frequency dependent spectra are summarized in Fig. 6. In the measurement of a bulk droplet of IL, two Mo wires with $150 \mu \mathrm{m}$ diameter were held at $1 \mathrm{~mm}$ distance each other in the droplet. AC impedance measurements of bridge-shaped specimens were
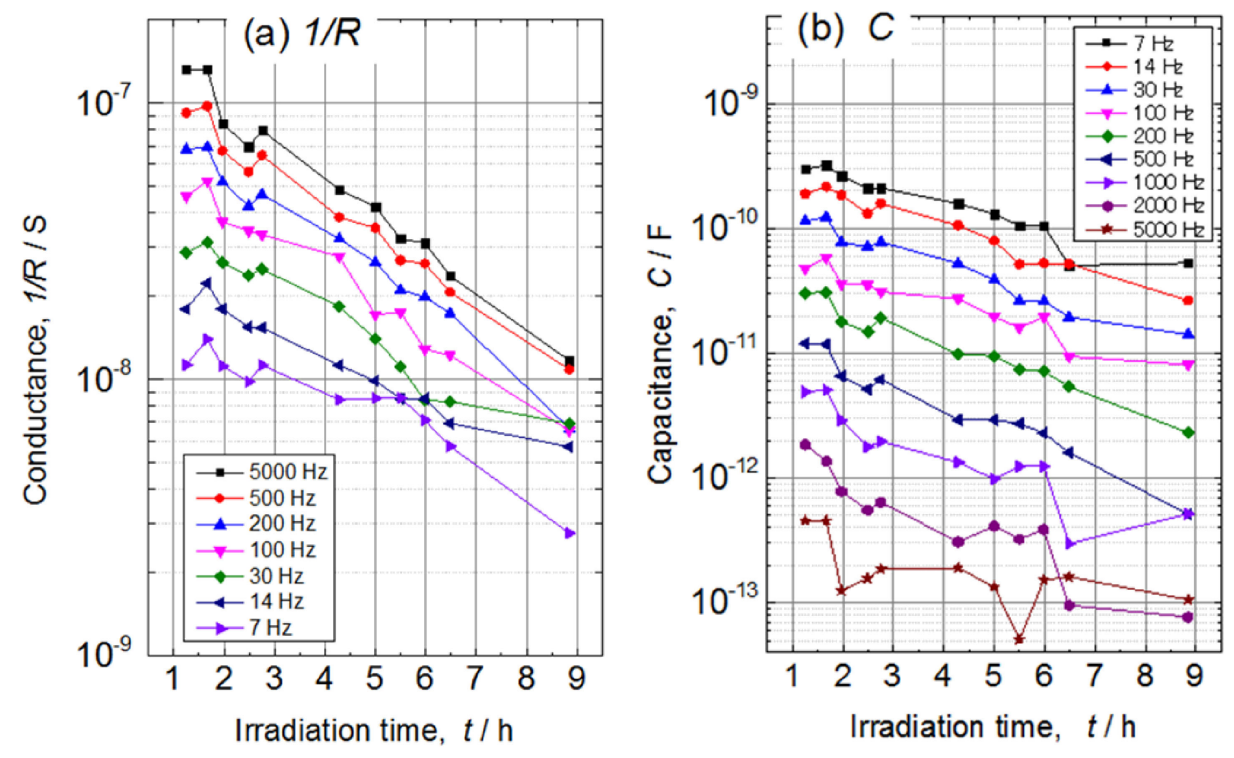

Fig. 4 Time evolution of conductance $(1 / \mathrm{R})$ and capacitance $(\mathrm{C})$ of the IL bridge under electron irradiation measured at different frequencies. 

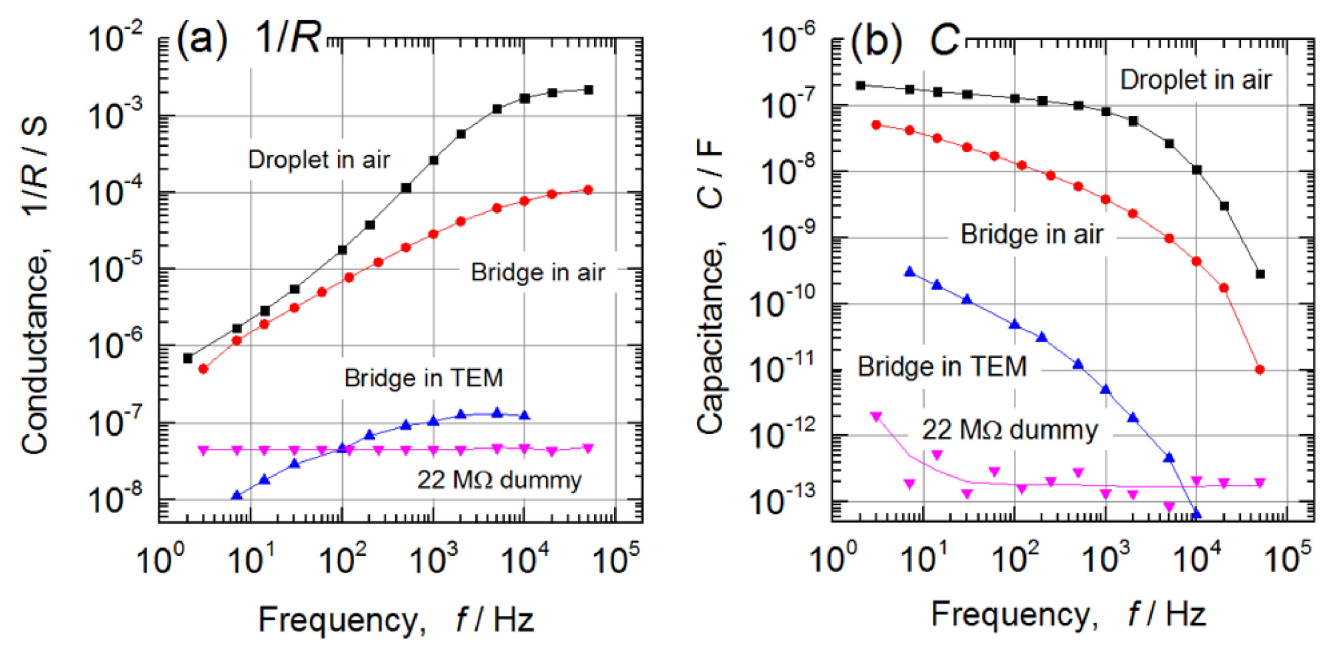

Fig. 6 Comparison of spectra of IL specimens; frequency dependent conductance (1/R) and capacitance (C) spectra of a bulk IL droplet, an IL bridge prepared in air, and an IL bridge experienced $1.3 \mathrm{~h}$ electron irradiation in a TEM. Spectra obtained with a $22 \mathrm{M} \Omega$ dummy specimen are also presented. Lines are guides to the eye.
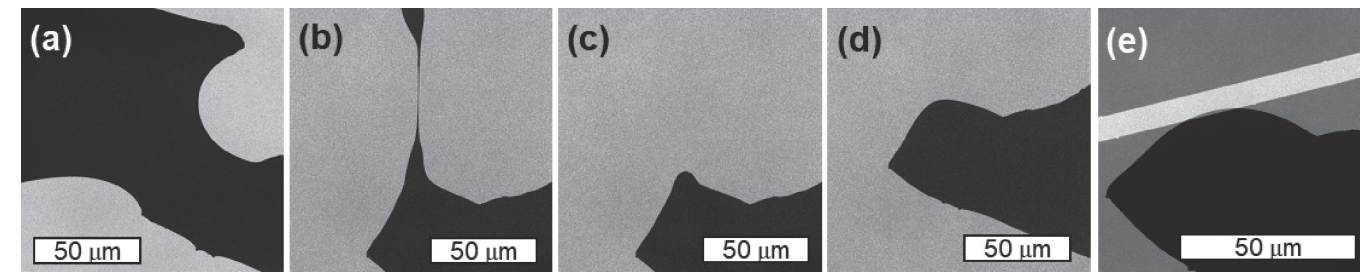

Fig. 7 Images of a breaking IL bridge. Rounding process of the IL surface occurred in 4 min. is shown in (b) to (d). Positioning of interference fringes for holography is shown in (e).

performed in air as well as in vacuum in the TEM. The displayed spectra of the IL bridge specimen in TEM (marked in blue) is limited between $7 \mathrm{~Hz}$ and $10 \mathrm{kHz}$ because of the poorer $\mathrm{S} / \mathrm{N}$ ratio outside of this range, which resulted from low conductance of the specimen in the low frequency side and its small capacitance in the high frequency side. Differences in the spectra of respective IL specimens will be discussed later in 4.1. Besides IL specimens, a resistor (an electronic circuit component) of $22 \mathrm{M} \Omega$ resistance was mounted on the specimen holder for the TEM as a dummy specimen. Flat spectrum of conductance up to $50 \mathrm{kHz}$ of this $22 \mathrm{M} \Omega$ dummy in Fig. 6(a) implies the reliability of the measurement of specimens with similar or larger value of conductance.

\subsection{Electron holography}

After completing the repeated AC impedance measurements, Mo probe tips were moved apart to break the IL bridge. Series of images during this breaking process are shown in Fig. 7. The surface of the broken IL bridge became rounded in time; it took four minutes between Fig. 7(b) and (d). The interference fringes for electron holography were positioned as in Fig. 7(e). The base of the probe tip bearing the IL was electrically grounded during the entire holography experiment.

A hologram and a reconstructed phase image of the broken IL bridge are shown in Fig. 8(a) and (b) respectively. In the hologram, Fig. 8(a), $5.3 \mu \mathrm{m}$-wide area is covered by 134 periods of interference fringes. For comparison, a hologram and a phase image of an IL specimen that had been recorded

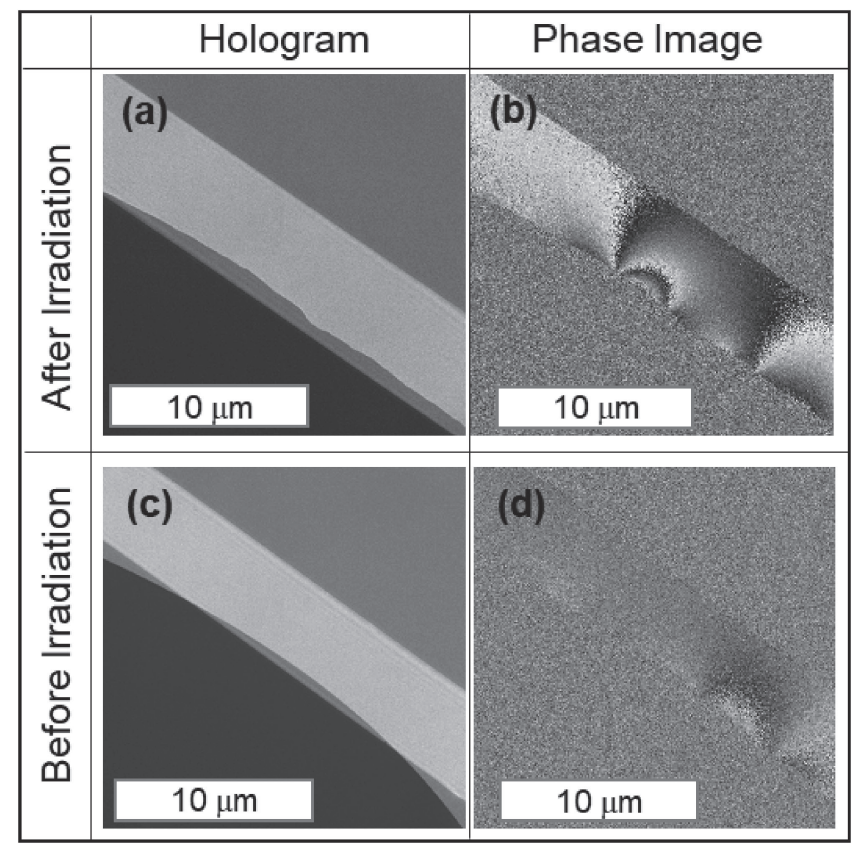

Fig. 8 Holograms and reconstructed phase images of a surface of IL specimen before and after electron irradiation.

prior to the bridge formation are shown in Fig. 8(c) and (d). The value mapped on a phase image is proportional to the value of the electric potential integrated along the trajectory of each electron contributed to the hologram. ${ }^{22}$ Phase contours enhanced after the continued electron irradiation, Fig. 8(b), implies electrostatic charging of the IL specimen, 

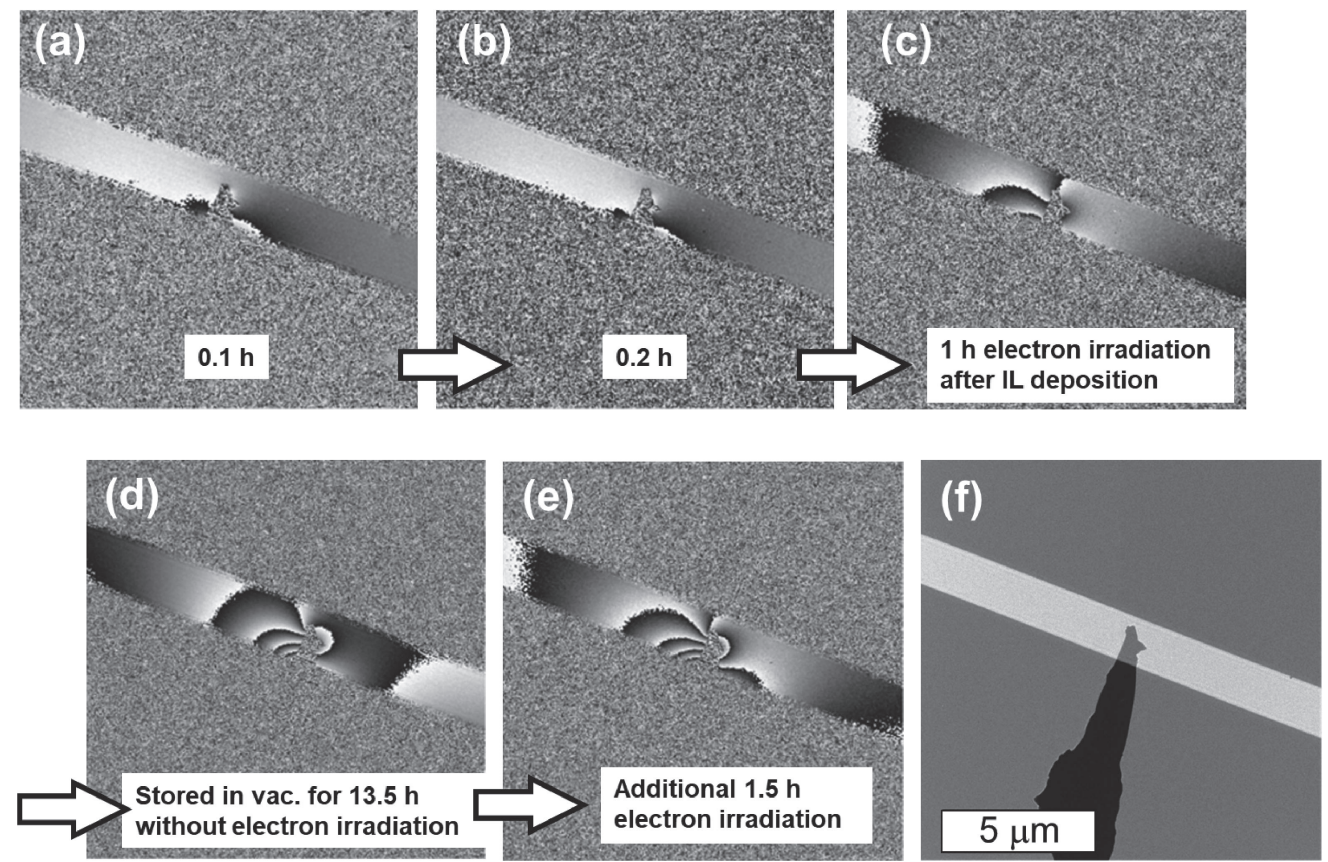

Fig. 9 Time-evolution of phase images, (a) to (e), around an IL-coated Mo tip under electron exposure. The shape of the tip is shown in the hologram, (f).

which is consistent with the decreased electrical conductance of the IL bridge observed via AC impedance measurement.

Results of another holography experiment are summarized in Fig. 9. A thinner Mo probe tip fabricated by focused ion beam was immersed into an IL droplet in a TEM to make a coating of IL around the tip, then the tip was retracted from the droplet and successively imaged during continued electron irradiation. The higher density of increasing phase contour than in Fig. 8 implies that the charging, or in other words, loss of conductivity of the IL coating under electron irradiation was more pronounced with the smaller specimen in Fig. 9.

The image with dense contours in Fig. 9(d) was recorded after $13.5 \mathrm{~h}$ of storage in vacuum after the initial electron irradiation had been terminated. This significantly increased contours imply that the loss of conductivity of the IL coating proceeded substantially during the period without electron irradiation.

\section{Discussion}

\subsection{Frequency dependence of $1 / R$ and $C$}

Comparing frequency dependent spectra of conductance and capacitance in Fig. 6, none of the plots overlap nor even have parallel slopes one another. Different slopes, i.e. different frequency responses, of conductance spectra of "Droplet in air" and "Bridge in air" recognized in Fig. 6(a) might seem strange for specimens both prepared with the same IL material and measured in the same atmosphere. A possible origin of the different slopes will be discussed by modeling the specimen with equivalent circuits.

A simple equivalent circuit for a specimen containing movable electric charges is shown in Fig. 10(a), where resistance and capacitance of the circuit elements are assumed to be frequency-independent constants. The speci- men material modeled in Fig. 10(a) consists of two types of regions; interface regions at the surface of both electrodes, and bulk liquid or "offshore" region apart from the electrodes. At low frequencies, since electric field from the electrode is screened by the charged interface layer, offshore charges do not respond to the externally applied voltage and thus do not contribute much to the current. At high frequencies polarity of applied voltage changes before the interface layer is well charged, which allows offshore charges also to contribute to the current. This is an illustration of the increasing conductance, $1 / \mathrm{R}$, with frequency. Broken lines in Fig. 10(c) were spectra calculated using the type of circuits as in Fig. 10(a), in which increasing $1 / \mathrm{R}$ and decreasing $\mathrm{C}$ both with frequency are reproduced.

The measured slope of increasing $1 / \mathrm{R}$ in Fig. 10(c) is gentler than the slopes calculated with circuits in Fig. 10(a). This discrepancy can be understood by the non-uniformity of the current channel in the real specimen. The specimen is not a material filled in a pair of parallel plates, and thus flow lines of electric current between electrodes have variety of lengths. Circuit parameters of interface layer may also vary depending on the roughness of the electrode surface. Taking these non-uniformities into account, the realistic equivalent circuit becomes a parallel combination of channels with different parameters. By means of combination of only two channels as shown in Fig. 10(b), the measured spectrum of $1 / \mathrm{R}$ was fitted with an improved slope as shown by the solid line in Fig. 10(c). Parameters of this equivalent circuit are summarized in Table 1. While the values of these parameters determined for the very limited number of current channels do not have physical meaning directly connected to the real specimen, the simulated curve in Fig. 10(c) qualitatively demonstrates the effect of geometrical inhomogeneity among the current paths on the slope of the $1 / R$ spectrum. 
(a) Interface and bulk liquid

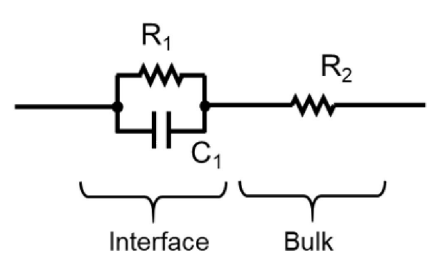

(b) Parallel channels

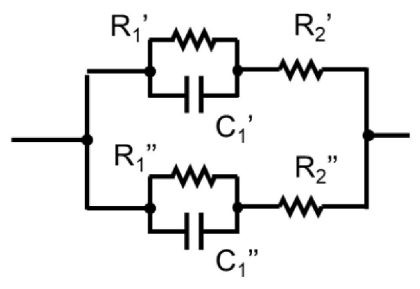

(c) Fitted spectra of a bulk IL measurement

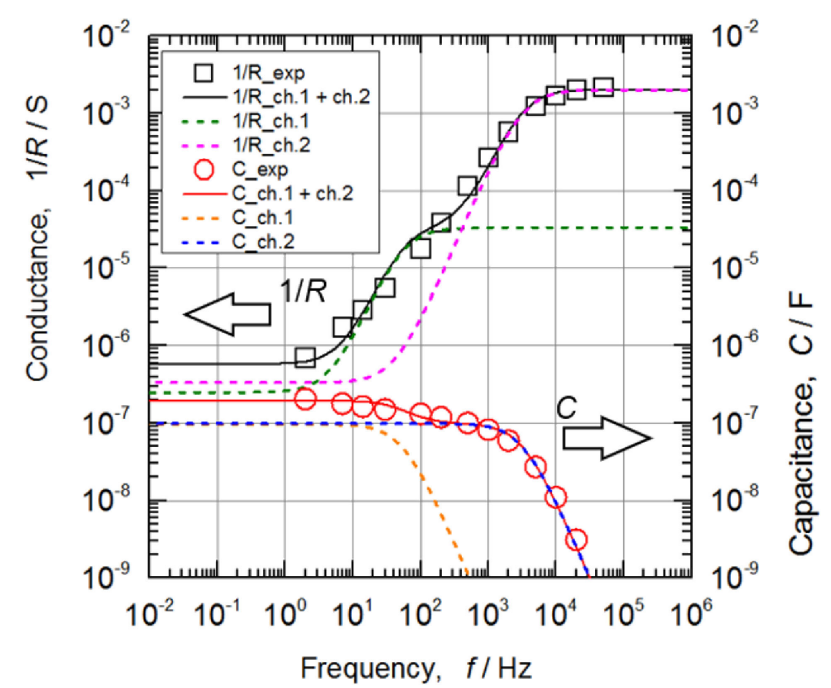

Fig. 10 Reproducing measured spectra with model calculations. (a) Equivalent circuit for a model of an IL specimen between metallic electrodes consisting of interface and bulk regions. (b) Equivalent circuit for a model of an IL specimen consisting of two parallel current channels. (c) Experimental data (adopted from "Droplet in air" in Fig. 6) and fitted spectra using a parallel channel model with two channels.

Table 1 Parameters of an equivalent circuit.

\begin{tabular}{lrrl}
\hline & $R_{1} / \mathrm{M} \Omega$ & $C_{1} / \mathrm{nF}$ & $R_{2} / \mathrm{k} \Omega$ \\
\hline Channel 1 & 4.0 & 84.6 & 40 \\
Channel 2 & 3.0 & 105 & 0.5 \\
\hline
\end{tabular}

Much gentler slope of increase in $1 / \mathrm{R}$ with frequency is observed in Fig. 6(a) with IL bridge specimen in air. Recalling that the supports for the IL bridge specimen are surfaces of cut Mo wires facing ca. $20 \mu \mathrm{m}$ apart, variety of surface roughness and lengths of current channels may well be more pronounced than in the case of bulk IL droplet, which should be described with an equivalent circuit with more current channels. The contribution of many parallel channels may even quantitatively account for the reduced steepness of the slope. The mechanism based on the geometry of the current channels described so far may be the primary reason for the difference in spectra of a bulk IL droplet and that of an IL bridge both measured in air, because no different frequency responses, or different dielectric dispersions, need to be assumed a priori to the IL specimens of the same material.

The spectra of $1 / \mathrm{R}$ and $\mathrm{C}$ for an IL bridge measured in a TEM are quite different from those measured in air as shown in Fig. 6. Firstly, conductance $(1 / R)$ and capacitance (C) both are smaller by more than two orders of magnitude. Secondly, spectrum of conductance showed saturation with increasing frequency above $2 \mathrm{kHz}$.

We were not able to identify the reason for the big discrepancies between spectra of IL bridges measured in air and in a TEM. As has been mentioned with Fig. 2, the bridge specimen was exposed to electron beam about an hour before the AC impedance measurements. This electron irradiation may have degraded conductivity of the IL specimen. Another possible cause is changing water content in vacuum. Remarkable increase of conductivity by addition of water has been reported for a type of IL. ${ }^{25)}$ If the IL used in the present experiment had similar nature, the loss of dissolved water in vacuum of the TEM may have resulted in decreased conductance of the specimen.

Since bridge specimen in air and in TEM must be similar in a sense that they both have similar geometry of current channels, it may not be suitable to explain the saturation of $1 / \mathrm{R}$ of the bridge in a TEM as the geometry-governed effect. This saturation should be understood as a change in frequency response of the material (i.e. loss of fast response of ionic motion) under electron irradiation, which is consistent with the pronounced decay time of $1 / \mathrm{R}$ at higher frequencies shown in Fig. 5.

\subsection{Applicability of impedance measurement to micro- scopic specimens}

In the present experiment we had to measure IL bridge specimens which were larger than common field of view of a TEM, and reconstructed phase images in Fig. 8 of electron holography contained only a limited part of the specimen. This is because the IL specimen exhibited small conductance and we had to increase the width of specimen to run current high enough to obtain sufficient $\mathrm{S} / \mathrm{N}$ ratio.

It is worthwhile to estimate the applicability of $\mathrm{AC}$ impedance measurement to microscopic specimens. Among superionic conductors, conductivity as high as $12 \mathrm{mS} / \mathrm{cm}$ at room temperature has been reported with $\mathrm{Li}_{10} \mathrm{GeP}_{2} \mathrm{~S}_{12}$ solid electrolyte. ${ }^{26)}$ If a TEM specimen of this material is fabricated by a FIB as thin as $150 \mathrm{~nm}$ with a dimension, $2 \mu \mathrm{m} \times 2 \mu \mathrm{m}$ (most of this area can be covered by interference fringes for electron holography), the resistance across the specimen is estimated to be $5.6 \mathrm{M} \Omega$, assuming that the material is free from irradiation damage and good ohmic contacts can be established with electrodes. A neat spectrum obtained with a $22 \mathrm{M} \Omega$ dummy specimen in Fig. 6 assures that the $\mathrm{AC}$ 
impedance measurement of this microscopic specimen with lower impedance $(5.6 \mathrm{M} \Omega)$ can be performed with sufficient $\mathrm{S} / \mathrm{N}$ ratio.

\subsection{Change in conductance of IL during vacuum storage}

A decrease of conductance in an IL specimen during vacuum storage was suggested by Fig. 9(d) obtained after the initial electron irradiation had been terminated. Similar loss of conductance during an interval of electron irradiation has not been observed in AC impedance measurements of $50 \mu \mathrm{m}$-thick IL bridges. The loss of conductance observed via electron holography on the FIB-fabricated thin probe may be a phenomenon only in the surface region of the IL coating. Detection of the type of change in a small volume remains as a future issue to be challenged with an improved sensitivity of the impedance measurement.

\section{Summary}

Using a specimen holder with a movable probe, an IL specimen that bridged two electrodes was prepared in the field of view of a TEM. The AC electrical impedance of the IL bridge was measured in the TEM in the frequency range from $3 \mathrm{~Hz}$ to $100 \mathrm{kHz}$.

Repeated impedance measurements revealed that the conductance $(1 / \mathrm{R})$ and capacitance $(\mathrm{C})$ of the IL bridge decreased with continued electron irradiation in the TEM. Characteristic decay time of $1 / \mathrm{R}$ was shorter at higher measurement frequencies, implying that the speed of the ionic motion in the IL became slower by the electron irradiation.

Distribution of electric potential around an IL specimen was characterized by electron holography before and after the electron irradiation in a TEM continued for $9.2 \mathrm{~h}$. Increased contours of electric potential were observed in a reconstructed phase image obtained after the electron irradiation, which is consistent with the loss of conductivity observed via $\mathrm{AC}$ impedance measurement.

For materials with sufficiently high conductivities such as $\mathrm{Li}_{10} \mathrm{GeP}_{2} \mathrm{~S}_{12}$ solid electrolyte, AC impedance measurement is expected to be capable of studying temporal response of carriers on a $150 \mathrm{~nm}$-thick microscopic specimen with $2 \mu \mathrm{m} \times 2 \mu \mathrm{m}$ area in a TEM, where potential distribution in space can be complementarily provided from electron holography.

\section{REFERENCES}

1) M. Nakanishi, G. Furusawa, K. Waki, Y. Hattori, T. Kamino, K. Sasaki, K. Kuroda and H. Saka: Mater. Trans. 48 (2007) 2572-2579.

2) M. Nakanishi, G. Furusawa, K. Waki, Y. Hattori, K. Sasaki, K. Fukunaga, T. Hirayama, H. Saka and K. Kuroda: Mater. Trans. 48 (2007) 2599-2605

3) T. Aiso, D. Shindo and T. Sato: Mater. Trans. 48 (2007) 2621-2625.

4) Z. Akase, D. Shindo, M. Inoue and A. Taniyama: Mater. Trans. 48 (2007) 2626-2630

5) J. Huang, X. Wang and X. Meng: Mater. Trans. 48 (2007) 2795-2798.

6) K. Yoshida, T. Nozaki, T. Hirayama and N. Tanaka: J. Electron Microsc. 56 (2007) 177-180.

7) C.-J. Choi, S.-M. Kang, H.-B. Hong, S.-H. Lee, J.-G. Kim, K.-S. Ahn and J.-W. Yoon: Mater. Trans. 51 (2010) 793-798.

8) K. Yamamoto, Y. Iriyama and T. Hirayama: Microscopy 66 (2017) 5061.

9) J.S. Wilkes and M.J. Zaworotko: Chem. Commun. 13 (1992) 965967.

10) S. Higashino, M. Miyake, H. Fujii, A. Takahashi, R. Kasada and T. Hirato: Mater. Trans. 59 (2018) 944-949.

11) S. Katsuyama, Y. Kusafuka and T. Tanakakase: Mater. Trans. 58 (2017) 1749-1753.

12) T. Uda, K. Tsuchimoto, H. Nakagawa, K. Murase, Y. Nose and Y. Awakura: Mater. Trans. 52 (2011) 2061-2066.

13) A. Lewandowski and A. Świderska-Mocek: J. Power Sources 194 (2009) 601-609.

14) M. Nakano, K. Shibuya, D. Okuyama, T. Hatano, S. Ono, M. Kawasaki, Y. Iwasa and Y. Tokura: Nature 487 (2012) 459-462.

15) S. Kuwabata, A. Kongkanand, D. Oyamatsu and T. Torimoto: Chem. Lett. 35 (2006) 600-601.

16) M. Shirai, T. Tanigaki, S. Aizawa, H.S. Park, T. Matsuda and D. Shindo: Ultramicroscopy 146 (2014) 125-129.

17) S.M.R. Niya and M. Hoorfar: J. Power Sources 240 (2013) 281-293.

18) N. Fukatsu, M. Hayashi, N. Kurita and T. Ohashi: Mater. Trans. 42 (2001) 1042-1047.

19) H. Katayama, Y.-C. Tay, A.S. Viloria, A. Nishikata and T. Tsuru: Mater. Trans. 38 (1997) 1089-1094.

20) A. Tonomura: Electron Holography, 2nd. ed., (Springer-Verlag, Heidelberg, 1999)

21) D. Shindo: Mater. Trans. 44 (2003) 2025-2034.

22) D. Shindo and Y. Murakami: J. Electron Microsc. 60 (2011) S225S237.

23) K. Harada, A. Tonomura, Y. Togawa, T. Akashi and T. Matsuda: Appl. Phys. Lett. 84 (2004) 3229-3231.

24) "Ionic Liquid for Electron Microscopy", Hitachi High-Technologies, https://www.hitachi-hightech.com/hfd/product_detail/?pn=em-i11000, (accessed 2019-08-28)

25) H. Abe, M. Aono and Y. Yoshimura: J. Chem. Chem. Eng. 6 (2012) 383-390.

26) N. Kamaya, K. Homma, Y. Yamakawa, M. Hirayama, R. Kanno, M. Yonemura, T. Kamiyama, Y. Kato, S. Hama, K. Kawamoto and A. Mitsui: Nat. Mater. 10 (2011) 682-686. 